\title{
MINLP OPTIMIZATION OF A CANTILEVER ROOF STRUCTURE
}

\author{
TOMAŽ ŽULA \& STOJAN KRAVANJA \\ Faculty of Civil Engineering, Transportation Engineering and Architecture, University of Maribor, Slovenia.
}

\begin{abstract}
This paper presents the simultaneous cost, standard sizes and rounded dimension optimization of a cantilever roof structure. Since the standard and rounded dimensions are proposed to be handled explicitly in the discrete type of the optimization, the mixed-integer non-linear programming (MINLP) is applied. The structure is built from the standard hot rolled steel I sections for columns, beams and struts, and from the reinforced concrete bases. The MINLP optimization model of the cantilever roof structure is developed. The model comprises the objective function of the structure's self-manufacturing costs and the design, resistance and dimensioning (in)equality constraints. The latter are defined in accordance with the Eurocode 2, 3 and 7 specifications. The modified outer-approximation/equalityrelaxation (OA/ER) algorithm is used. The MINLP optimization of a cantilever roof structure for a small football stadium near the city of Maribor in Slovenia is presented at the end of the paper. The obtained optimal result includes the minimal production costs of the structure, the optimal standard sections of steel elements and the optimal rounded dimensions of the concrete bases.
\end{abstract}

Keywords: cost optimization, discrete optimization, mixed-integer non-linear programming, MINLP, roof structure.

\section{INTRODUCTION}

Recently, many different optimization approaches for structural optimization have been proposed. Cicconi et al. [1] have introduced virtual prototyping tools and the genetic algorithm to reduce the weight and cost of steel structures. Kalanta et al. [2] have reported the discrete optimization of steel bar structures using the branch and bound method. Hasançeb [3] has introduced the sizing optimization of steel frames by the evolution strategy method. Mela and Heinisuo [4] have applied the particle swarm optimization method for the weight minimization and the minimum cost optimization of welded high strength steel beams. Zhang et al. [5] have reported the optimization of long-span portal frame under dynamic wind loads using a surrogate-assisted evolutionary algorithm and Van Mellaert et al. [6] have proposed the discrete sizing optimization of frame structures using the mixed-integer linear programing (MILP).

This paper examines the simultaneous cost, standard sizes and rounded dimension optimization of a cantilever roof structure. This type of the structure can be used for car parking roofs, small bus stations, small stadiums, etc. In order to obtain a real roof structure, standard and rounded dimensions are proposed to be handled explicitly in the model that lead to perform the discrete type of the optimization. The optimization is calculated using the mixed-integer non-linear programming approach (MINLP). The MINLP is a combined continuous-discrete optimization method. It performs the continuous optimization of parameters (production costs, masses, internal forces, deflections), the discrete optimization of discrete sizes (the standard steel sections of columns, beams and struts) as well as the rounded dimension optimization of the reinforced concrete bases (the length and width of the bases are automatically rounded on whole $10 \mathrm{~cm}$ ).

The MINLP optimization model of the cantilever roof structure is developed. The objective function of the self-manufacturing costs of the structure is defined and subjected to the design, structural analysis and dimensioning constraints. The dimensioning constraints are 
defined in accordance with Eurocode 3 [7] for steel, Eurocode 2 [8] for concrete and Eurocode 7 [9] for geotechnics. The ultimate and serviceability limit state conditions are in this way satisfied through the optimization.

This paper reports the approach for solving the MINLP optimization problem using the modified outer-approximation/equality-relaxation (OA/ER) algorithm, see Kravanja and Grossmann [10], and Kravanja et al. [11-12]. A single-phase MINLP optimization is proposed. The calculation begins with the continuous optimization of the structure. The first result is used as the starting point for the further second step, where the discrete optimization of standard sizes and rounded dimensions was executed.

\section{M I N L P OPTIMIZATION}

The optimization problem of the roof structure is non-convex and non-linear. Since handling the discrete alternatives of the structure requires executing the discrete type of the optimization, the mixed-integer non-linear programming (MINLP) is applied. The general MINLP optimization problem is defined as follows:

$$
\begin{gathered}
\min \quad \mathrm{z}=\mathbf{c}^{\mathrm{T}} \mathbf{y}+f(\mathbf{x}) \\
\text { subjected to: } \\
\mathbf{g}(\mathbf{x}) \leq \mathbf{0} \\
\mathbf{B y}+\mathbf{C} \mathbf{x} \leq \mathbf{b} \\
\mathrm{x} \in X=\left\{\mathrm{x} \in R^{n}: \mathrm{x}^{\mathrm{LO}} \leq \mathrm{x} \leq \mathrm{x}^{\mathrm{UP}}\right\} \\
\mathrm{y} \in Y=\{0,1\}^{\mathrm{m}}
\end{gathered}
$$

In the model formulation, $\mathbf{x}$ is a vector of continuous variables and $\mathbf{y}$ represents a vector of discrete mostly binary $0-1$ variables. While $\mathbf{c}^{\mathrm{T}} \mathbf{y}$ represents a linear fixed item, $f(\mathbf{x})$ stands for a dimension dependent item in the objective function $z$. The latter is subjected to equality and inequality constraints $\mathbf{g}(\mathbf{x})$ and to mixed linear (in)equality constraints $\mathbf{B y}+\mathbf{C x} \leq \mathbf{b}$. Functions $z$ and $\mathbf{g}(\mathbf{x})$ must be continuous and differentiable. At least one of them must be non-linear.

The MINLP optimization of the cantilever roof structure is calculated by the MINLP computer program MIPSYN [13] and [14]. The modified OA/ER algorithm and the single-phase MINLP optimization are applied. GAMS/CONOPT2 (generalized reduced-gradient method) by Drudd [15] is used to solve NLP problems, whilst GAMS/CPLEX [16] (branch and bound) is used to solve MILP problems.

The OA/ER algorithm contains of solving an alternative sequence of non-linear programming optimization subproblems (NLP) and mixed-integer linear programming master problems (MILP), Fig. 1. The first corresponds to the optimization of parameters for a building structure with a fixed standard dimensions, standard materials and rounded dimensions yields an upper bound to the objective to be minimized. The last mentioned involve a global linear approximation to the superstructure of alternatives in which a new standard sizes, standard materials and rounded dimensions are identified. When the problem is convex the search is terminated when the predicted lower bound exceeds the upper bound, otherwise it is terminated when the NLP solution can be improved no more. 


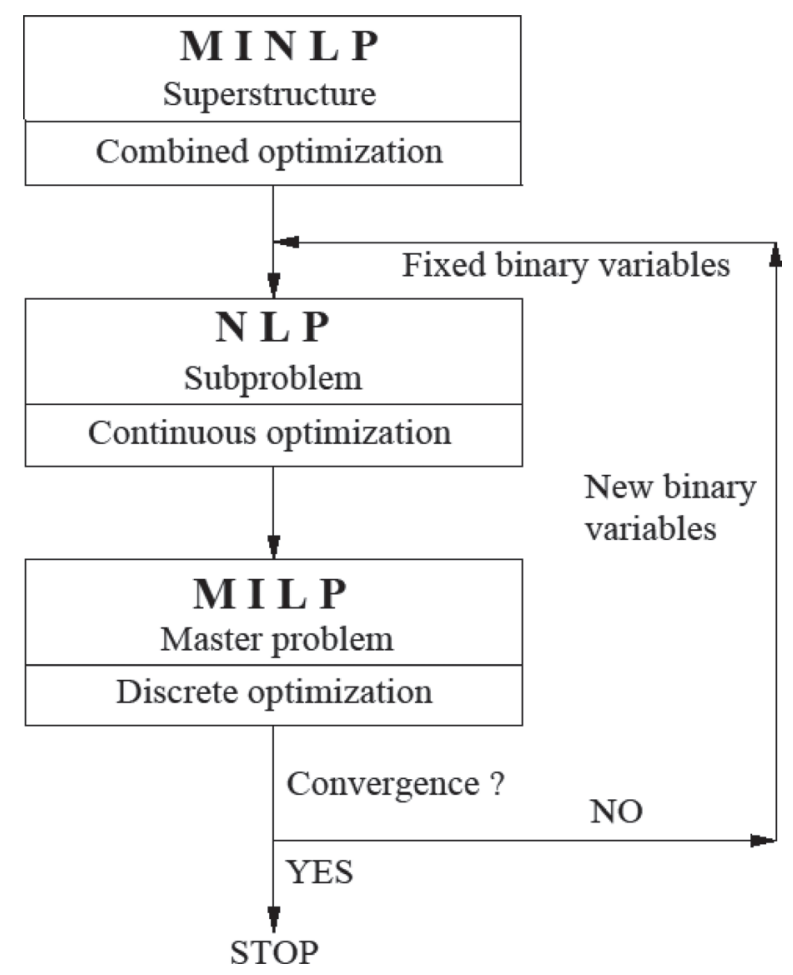

Figure 1: MINLP optimization program architecture.

\section{OPTIMIZATION MODEL CANROOF}

The cantilever roof structure is constructed from columns, beams, struts and bases, see Fig. 2. The columns, beams and struts are proposed to be built up from the standard hot rolled steel I section, while the bases are made from the reinforced concrete. Purlins which are mutually connected onto cantilever frames are not included in the optimization; the same holds with the vertical and horizontal bracing systems. The roof structure is defined as an MINLP superstructure, composed of various discrete alternatives, i.e. different standard sections for the steel elements and different rounded dimension alternatives for the concrete bases.

According to the above MINLP model formulation, the MINLP optimization model CANROOF (CANtilever ROOF structure) was developed. The model was modelled in GAMS (General Algebraic Modelling System) [17] environment. The model is consisted from input data, continuous and discrete binary variables, the structure's cost objective function, and from structural analysis constraints and logical constraints.

The input data comprise the sets for defining the discrete alternatives of standard dimensions and rounded dimensions, and scalars and parameters. Defined are $s, s \in S$, standard cross-section alternatives for columns, beams and struts separately and $r, r \in R$, rounded dimension alternatives for length and width of the concrete base.

The scalars in input data contain the global geometry of the cantilever roof: the length of the beam $L_{c r}$, the length of the strut $L_{s}$, the height of the column $h_{c}$, the over height of the frame 


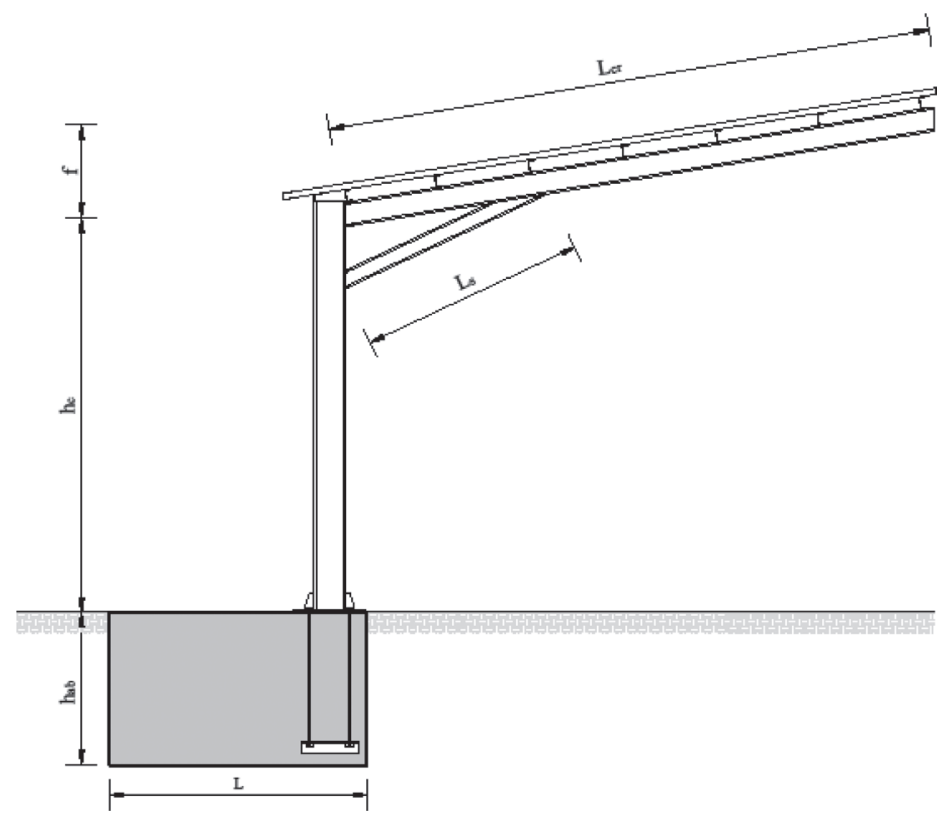

Figure 2: Cantilever roof structure.

beam $f$ and the height of the reinforced concrete base $h_{a b}$, the elastic modulus of the steel $E$, the shear modulus of the steel $G$, the yield strength of the steel $f_{y}$ the density of the steel $\rho_{s}$, the density of the reinforced concrete $\rho_{z}$, the variable load (snow $s$, the vertical wind $w_{v}$, the horizontal wind $w_{h}$ ), the partial safety factor for the permanent load $\gamma_{g}(1.35)$, the partial safety factor for the variable load $\gamma_{q}(1.50)$, the resistance partial safety factors $\gamma_{M 0}(1.0)$ and $\gamma_{M I}$ (1.0), the material and erection cost (price) of the concrete $C_{c}$, the material cost of the structural steel $C_{s}$, the material cost of the reinforcing steel $C_{r}$, the anti-corrosion resistant painting cost $C_{a c}$, the cost per steel connection $C_{s c}$, the construction pit (incl. excavation, removal, planning) $C_{p}$, the panelling cost (incl. material and erection) $C_{p l}$, etc.

The parameters in input data include the vectors of different discrete alternative values of the standard dimensions $q_{s}$ (standard sizes of IPE and HEA sections) as well as the discrete alternative values of the rounded dimensions $q_{r}$ (the length and width of the concrete base were proposed as being rounded up to whole $10 \mathrm{~cm}$ ).

The continuous variables contain the self-manufacturing costs of the cantilever roof structure COSTS, the overall breadths of the column, beam and strut $b$; the cross-sectional heights of the column, beam and strut $h$; the flange thicknesses of the column, beam and strut $t_{f}$; the web thicknesses of the column, beam and strut $t_{w}$; the cross-sections of the column, beam and strut $A$; the elastic section modules of the column and beam $W$; the design bending moments in the column and beam $M_{E d}$, the design axial forces in the column, beam and strut $N_{E d}$; the design shear forces in the column and beam $V_{E d}$, the vertical deflection of the cantilever roof structure $\delta$, the length of the base $L$ and the width of the base $b_{b}$.

The binary variables comprise binary variables $\mathbf{y}_{s}$ for $s, s \in S$, standard cross-section alternatives of columns, beams and struts separately, and binary variables $\mathbf{y}_{r}$ for $r, r \in R$, rounded dimension alternatives of length and width of the concrete base. 
The cost objective function represents the self-manufacturing costs of the material, anti-corrosion resistant painting, panelling, connections and assembling, see:

min: $\operatorname{COST}=C_{\mathrm{c}} \cdot V_{\mathrm{c}}+C_{\mathrm{s}} \cdot \rho_{\mathrm{s}} \cdot V_{\mathrm{s}}+C_{\mathrm{r}} \cdot \rho_{\mathrm{s}} \cdot V_{\mathrm{r}}+C_{\mathrm{ac}} \cdot A_{\mathrm{ac}}+C_{\mathrm{sc}}+0.15 \cdot C_{\mathrm{s}} \cdot \rho_{\mathrm{s}} \cdot V_{\mathrm{s}}+C_{\mathrm{p}} \cdot V_{\mathrm{p}}+C_{\mathrm{pl}} \cdot A_{\mathrm{pl}}$

where COST defines the self-manufacturing costs of the cantilever roof structure; $C_{\mathrm{c}}, C_{\mathrm{s}}, C_{\mathrm{r}}$, $C_{\mathrm{ac}}, C_{\mathrm{sc}}, C_{\mathrm{p}}$ and $C_{\mathrm{pl}}$ are the prices of the concrete and steel materials, of the anti-corrosion resistant painting, of the steel connections as well as the prices of the construction pit and of the panelling, see Table $1 . V_{\mathrm{c}}, V_{\mathrm{s}}, V_{\mathrm{r}}$ and $V_{\mathrm{p}}$ represent the volumes of the concrete base, the steel I sections, the reinforcing steel and of the excavation, respectively; $\rho_{\mathrm{s}}$ is the unit mass of the steel; $A_{\mathrm{ac}}$ is the exposed area of the steel members; and $A_{\mathrm{pl}}$ is the area of the panelling. Assembling costs of the steel structure is defined to be $15 \%$ of the calculated material costs.

The structural analysis constraints include the calculation of internal forces and deflections according to force method and the constraints for dimensioning. Only the main constraints are presented in the paper. The ultimate limit state constraints of structural elements (the Eurocode specifications) include eqns (2)-(11) and the serviceability limit state constraint comprise eqn (12).

The axial resistances of the steel members are checked by eqn (2), where $N_{E d}$ stands for the design axial force, $A$ represents the cross-section area of the steel member, $f_{y}$ is the yield strength of the steel and $\gamma_{M 0}$ is the resistance partial safety factor.

$$
N_{E d} \leq \frac{A \cdot f_{y}}{\gamma_{M 0}}
$$

The shear resistances of the members are verified by eqn (3), where $V_{E d}$ denotes the design shear force and $A_{v}$ is the effective shear area of the cross-section.

$$
V_{E d} \leq A_{v} \frac{f_{y}}{\sqrt{3}} \cdot \frac{1}{\gamma_{M 0}}
$$

The bending moment resistances of the members are determined by eqn (4). $M_{E d}$ represents the design bending moment and $W$ is the section modulus of the steel member.

$$
M_{E d} \leq \frac{W \cdot f_{y}}{\gamma_{M 0}}
$$

The compression/buckling resistances are defined by eqn (5), where $\chi$ is the reduction factor due to the flexural buckling and $\gamma_{M 1}$ is the resistance partial safety coefficient.

$$
N_{E d} \leq \chi \frac{A \cdot f_{y}}{\gamma_{M I}}
$$

The interaction between the compression and bending moment resistances is defined by eqn (6). 


$$
\frac{N_{E d}}{A \cdot f_{y} / \gamma_{M O}}+\frac{M_{E d}}{W \cdot f_{y} / \gamma_{M O}} \leq 1.0
$$

The interaction between the buckling and the lateral-torsional buckling resistances is checked by eqn (7), where $\chi_{L T}$ is the reduction factor due to the lateral-torsional buckling.

$$
\frac{N_{E d}}{\chi \cdot A \cdot f_{y} / \gamma_{M 1}}+\frac{M_{E d}}{\chi_{L T} \cdot W \cdot f_{y} / \gamma_{M 1}} \leq 1.0
$$

The bearing resistance failure in the ground under the reinforced concrete bases is checked by eqn (8). Here, $N_{E d}$ is the vertical design force and $M_{E d}$ is the design bending moment, which acts on the concrete base. $A_{b}, L$ and $b_{b}$ stand for the surface area, length and width of the base, respectively. While the surface area is calculated by eqn (9), the section modulus of the base $W_{b}$ is defined by eqn (10).

$$
\begin{gathered}
\sigma_{E d}=\frac{N_{E d}}{A_{b}}+\frac{M_{E d}}{W_{b}} \leq 200 \mathrm{kPa} \\
A_{b}=L \cdot b_{b} \\
W_{b}=\frac{b_{b} \cdot L^{2}}{6}
\end{gathered}
$$

The overturning resistance of the reinforced concrete base is defined by eqn (11), where $G$ is the self-weight of the reinforced concrete base, $F_{\text {tot }}$ is the total action force (the self-weight of the roof structure and the base plus snow plus wind), $e$ is the distance between the total force and the point of overturning (the right bottom base edge) and $\gamma$ is the safety factor $(\gamma=2.5)$.

$$
G \cdot \frac{L}{2} \geq F_{t o t} \cdot e \cdot \gamma
$$

The total vertical deflection $\delta$ of the cantilever roof structure is checked by eqn (12), where $L_{c r}$ is the span of the cantilever roof.

$$
\delta \leq \frac{2 \cdot L_{c r}}{200}
$$

The logical constraints determine the discrete values for standard dimensions $d^{\text {st }}$ (of the standard steel sections) and the discrete values for rounded dimensions $d^{r d}$ (of the concrete bases), see eqns (13)-(16). The standard dimensions and rounded dimensions are calculated as a scalar product between the vector of discrete alternatives $q$ and the vector of associated binary variables $y$. Only one discrete value is selected for the standard dimension or for the rounded dimension, since the sum of the binary variables $y$ has to be equal to 1 , see eqn (14) and eqn (16).

$$
d^{s t}=\sum_{s \in S} q_{s} y_{s}^{s t} \quad s \in S
$$




$$
\begin{gathered}
\sum_{s \in S} y_{s}^{s t}=1 \quad s \in S \\
d^{r d}=\sum_{r \in R} q_{r} y_{r}^{r d} \quad r \in R \\
\sum_{r \in R} y_{r}^{r d}=1 \quad r \in R
\end{gathered}
$$

\section{NUMERICAL EXAMPLE}

The numerical example shows the simultaneous cost, standard and rounded dimension optimization of a steel cantilever roof structure for a small football stadium near the city of Maribor, Slovenia. A number of variants of the roof structure were considered and calculated. While the optimization of the first variant was already reported by Žula and Kravanja [18], the presented variant includes a bit bigger roof structure than that in [18] (16.5 metres long, $4.0 \mathrm{~m}$ high and $4.6 \mathrm{~m}$ wide). The treated steel structure is now $16.5 \mathrm{~m}$ long, $4.6 \mathrm{~m}$ high and 6.0 m wide, see Fig. 3.

The structure consists from four equal cantilever frames with the intermediate distances of $5.5 \mathrm{~m}$, clamped onto four reinforced equal concrete bases. Each reinforced concrete base is $2.0 \mathrm{~m}$ deep, placed behind the existed concrete seat structure. The in-plane length and width of the base is treated in the optimization as variables. Steel S 355 and concrete C25/30 were used. The structure is exposed to the combined effect of the permanent uniform load (selfweight) and the uniformly distributed variable load $q$ (snow $s=1.25 \mathrm{kN} / \mathrm{m}^{2}$ and wind $w=0.25$ $\mathrm{kN} / \mathrm{m}^{2}$ ). The design loads are defined: $1.35 \cdot g+1.50 \cdot \mathrm{s}+1.50 \cdot 0.6 \cdot \mathrm{w}$ for the ultimate limit states, and $1.00 \cdot g+1.00 \cdot s+1.00 \cdot 0.6 \cdot w$ for the serviceability limit states.

The MINLP optimization model CANROOF of the structure is applied. The model comprises the structure's cost objective function, for which the material and labour costs (prices) are defined and shown in Table 1.
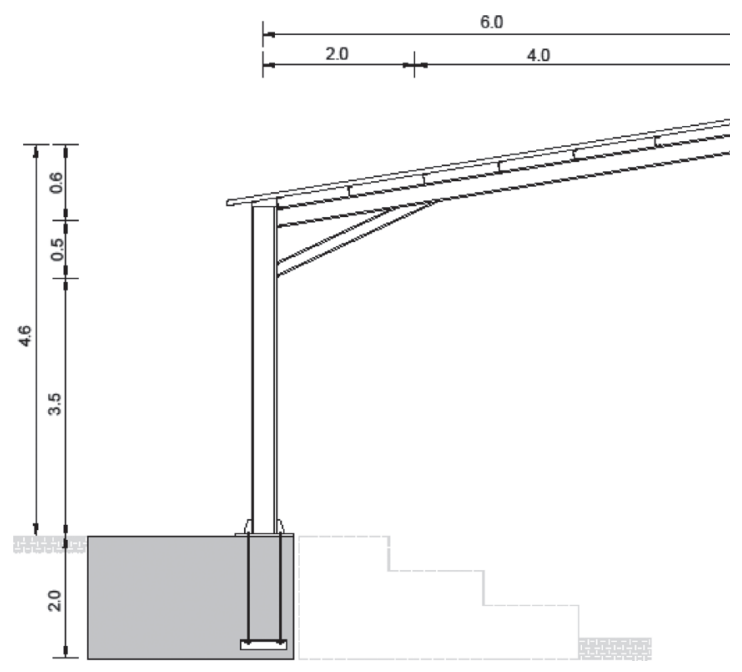

Figure 3: Global dimensions of the cantilever roof structure. 
Table 1: Material and labour costs (prices).

\begin{tabular}{lll}
\hline$C_{\mathrm{c}}$ Concrete C 25/30 & 125.0 & $\mathrm{EUR} / \mathrm{m}^{3}$ \\
$C_{\mathrm{s}}$ Structural steel S 355 & 1.25 & $\mathrm{EUR} / \mathrm{kg}$ \\
$C_{\mathrm{r}}$ Reinforcing steel B 500 & 1.30 & $\mathrm{EUR} / \mathrm{kg}$ \\
$C_{\mathrm{ac}}$ Anti-corrosion resistant painting & 20.0 & $\mathrm{EUR} / \mathrm{m}^{2}$ \\
$C_{\mathrm{sc}}$ Steel connection & 300.0 & $\mathrm{EUR}$ \\
$C_{p}$ Construction pit & 10.0 & $\mathrm{EUR} / \mathrm{m}^{3}$ \\
$C_{\mathrm{pl}}$ Panelling & 5.0 & $\mathrm{EUR} / \mathrm{m}^{2}$ \\
\hline
\end{tabular}

The superstructure was generated in which all possible cantilever structure alternatives were embedded within a combination between 24 different standard HEA steel sections (from HEA 100 to HEA 1000) for the column, 18 different standard IPE steel sections (from IPE 80 to IPE 600) for the beam and strut separately, 94 various discrete alternatives for rounding up the length of the reinforced concrete base (from $70 \mathrm{~cm}$ to $1000 \mathrm{~cm}$ ) and 94 various discrete alternatives for rounding up the width of the reinforced concrete base (from $70 \mathrm{~cm}$ to $1000 \mathrm{~cm}$ ). In this way, the superstructure of alternatives includes $24 \cdot 18 \cdot 18 \cdot 94.94=$ $6.8708736 \cdot 10^{7}$ different solutions of the discrete variables.

The single-phase MINLP optimization is applied. The procedure begins with the continuous NLP optimization of the structure (the initialization), where the standard and rounded dimensions are temporary relaxed. The optimal structure's self-manufacturing costs of 20,579€ (for all four cantilever frames) are obtained using the relaxed/continuous standard and rounded dimensions, see the 1st NLP in Table 2. This result is used as the starting point for the discrete standard and rounded dimension optimization. The convergence of the modified OA/ER algorithm is shown in Table 2. At this level, the binary variables $y_{s}$ of the standard sizes for column, beam and strut, and the binary variables $y_{r}$ of the rounded dimensions for length and width of

Table 2: Convergence of the modified OA/ER algorithm.

\begin{tabular}{|c|c|c|c|c|c|c|}
\hline \multirow{2}{*}{$\begin{array}{l}\text { MINLP } \\
\text { Iteration }\end{array}$} & \multirow{2}{*}{$\begin{array}{c}\text { MINLP } \\
\text { Sub-phase }\end{array}$} & \multirow[b]{2}{*}{ Costs $[€]$} & \multicolumn{3}{|c|}{$\begin{array}{l}\text { Cross-section area }\left[\mathrm{cm}^{2}\right] / \\
\text { Standard steel section }\end{array}$} & \multirow{2}{*}{$\begin{array}{c}\text { Base area } \\
{\left[\mathrm{m}^{2}\right]} \\
\mathbf{A}_{\mathrm{b}}=\mathbf{L} \cdot \mathbf{b}_{\mathrm{b}}\end{array}$} \\
\hline & & & Column & Beam & Strut & \\
\hline \multicolumn{7}{|c|}{ Continuous optimization } \\
\hline 1. & 1. NLP & 20,579 & 174.99 & 55.80 & 37.09 & 8.70 \\
\hline \multicolumn{7}{|c|}{ Discrete standard and rounded dimension optimization } \\
\hline \multirow{2}{*}{2.} & 1. MILP & 21,077 & $178.00 /$ & $62.60 /$ & $45.90 /$ & 8.80 \\
\hline & 2. NLP & 21,077 & HEA 450 & IPE 330 & IPE 270 & $4.40 \cdot 2.00$ \\
\hline \multirow{2}{*}{3.} & 2. MILP & 20,924 & $178.00 /$ & $62.60 /$ & $33.40 /$ & 8.80 \\
\hline & 3. NLP & 20,924 & HEA 450 & IPE 330 & IPE 220 & $4.40 \cdot 2.00$ \\
\hline \multirow{2}{*}{4.} & 3. MILP & 21,345 & $178.00 /$ & $62.60 /$ & $45.90 /$ & 9.03 \\
\hline & 4. NLP & 21,345 & HEA 450 & IPE 330 & IPE 270 & $4.30 \cdot 2.10$ \\
\hline
\end{tabular}




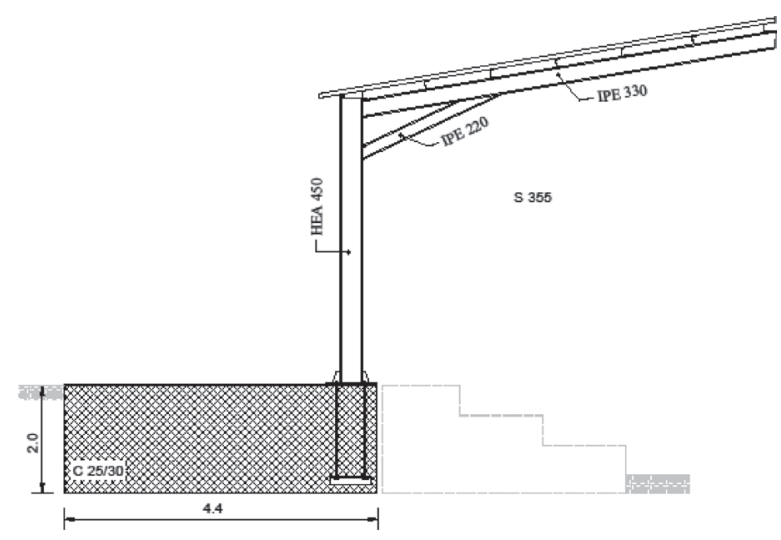

Figure 4: Optimum design of the cantilever roof structure.

the base are included in the optimization. The same holds with the logical constraints for discrete decisions. The final structure's minimal self-manufacturing costs of $20,924 €$ is obtained at the 3rd main MINLP iteration (the selling price is approx. 2.5 times higher than these production costs). The optimal solution also contains the calculated optimal standard sections of column (HEA 450), beam (IPE 330), strut (IPE 220) and the calculated rounded dimensions of the reinforced concrete base, i.e. the length of $4.40 \mathrm{~m}$ and the width of $2.00 \mathrm{~m}$, see Fig. 4 .

\section{CONCLUSIONS}

The paper deals with the cost, standard sizes and rounded dimension optimization of a cantilever roof structure, which can be made for car parking roofs, small bus stations, small stadiums, etc. The discrete optimization of the structure is performed with the mixed-integer non-linear programming, MINLP. The MINLP optimization model is developed. The cost objective function of the structure is subjected to the structural analysis and dimensioning constraints. The modified OA/ER algorithm and the MINLP computer package MIPSYN are applied. The MINLP optimization of the cantilever roof structure for a small football stadium is presented at the end of the paper. The optimal solution comprises the minimal self-manufacturing costs of the structure, standard sections of steel elements and rounded dimensions of the concrete bases.

Such applications of different optimization techniques in the engineering practice represent a novel and advantageous manner. The optimized self-manufacturing costs of the structure in the paper was found to be more than $20 \%$ lower than the costs of the structure, obtained by the classical structural analysis. The example clearly shows the efficiency of the proposed MINLP optimization approach.

\section{ACKNOWLEDGEMENTS}

The authors are grateful for the support of funds from the Slovenian Research Agency (program P2-0129).

\section{REFERENCES}

[1] Cicconi, P., Germani, M., Bondi, S., Zuliani, A. \& Cagnacci, E., A design methodology to support the optimization of steel structures. Procedia CIRP, 50, pp. 58-64, 2016. https://doi.org/10.1016/j.procir.2016.05.030 
[2] Kalanta, S., Atkočiunas, J. \& Venskus, A., Discrete optimization problems of the steel bar structures. Engineering Structures, 31(6), pp. 1298-1304, 2009. https://doi. org/10.1016/j.engstruct.2009.01.004

[3] Hasançeb, O., Cost efficiency analyses of steel frameworks for economical design of multistorey buildings. JCSR, 128, pp. 380-396, 2017. https://doi.org/10.1016/j.jcsr.2016.09.002

[4] Mela, K. \& Heinisuo, M., Weight and cost optimization of welded high strength steel beams. Engineering Structures, 79, pp. 354-364, 2014. https://doi.org/10.1016/j.engstruct.2014.08.028

[5] Zhang, Z., Pan, J., Fu, J., Singh, H.K., Pi, Y.L., Wu, J. \& Rao, R., Optimization of long span portal frames using spatially distributed surrogates. Steel and Composite Structures, 24, pp. 227-237, 2017.

[6] Van Mellaert, R., Mela, K., Tiainen, T., Heinisuo, M., Lombaert, G. Schevenels, M., Mixed-integer linear programming approach for global discrete sizing optimization of frame structures. Structural and Multidisciplinary Optimization, 57(2), pp. 579-593, 2018. https://doi.org/10.1007/s00158-017-1770-9

[7] Eurocode 3. Design of steel structures, European Committee for Standardization, Brussels 2005.

[8] Eurocode 2. Design of concrete structures, European Committee for Standardization, Brussels 2004.

[9] Eurocode 7. Geotechnical design, European Committee for Standardization, Brussels 2004.

[10] Kravanja, Z. \& Grossmann, I.E., New Developments and Capabilities in PROSYN-An Automated Topology and Parameter Process Synthesizer. Computers \& Chemical Engineering, 18(11-12), pp. 1097-1114, 1994. https://doi.org/10.1016/ s0098-1354(94)85027-5

[11] Kravanja, S., Kravanja, Z. \& Bedenik, B.S., The MINLP optimization approach to structural synthesis. Part I: A general view on simultaneous topology and parameter optimization. International Journal for Numerical Methods in Engineering, 43(2), pp. 263-292, 1998. https://doi.org/10.1002/(sici)1097-0207(19980930)43:2<263::aid-nme412>3.0.co;2-u

[12] Kravanja, S., Kravanja, Z. \& Bedenik, B.S., The MINLP optimization approach to structural synthesis. Part II: Simultaneous topology, parameter and standard dimension optimization by the use of the Linked two-phase MINLP strategy. International Journal for Numerical Methods in Engineering, 43(2), pp. 293-328, 1998. https://doi. org/10.1002/(sici)1097-0207(19980930)43:2<293::aid-nme413>3.3.co;2-f

[13] Kravanja, S., Soršak, A. \& Kravanja, Z., Efficient multilevel MINLP strategies for solving large combinatorial problems in engineering. Optimization and Engineering, 4(1/2), pp. 97-151, 2003. https://doi.org/10.1023/a:1021812414215

[14] Kravanja Z., Challenges in sustainable integrated process synthesis and the capabilities of an MINLP process synthesizer, MipSyn. Computers \& Chemical Engineering, 34(11), pp. 1831-1848, 2010. https://doi.org/10.1016/j.compchemeng.2010.04.017

[15] Drudd, A.S., CONOPT-A Large-Scale GRG Code. ORSA Journal on Computing, 6(2), pp. 207-216, 1994. https://doi.org/10.1287/ijoc.6.2.207

[16] CPLEX 11 User's Manuals, ILOG inc., 2007.

[17] Brooke, A., Kendrick, D. \& Meeraus, A., GAMS - A User's Guide, Scientific Press, Redwood City, CA, 1988.

[18] Žula, T. \& Kravanja, S., Cost optimization of a cantilever roof structure for the small football stadium in Limbuš. WIT transactions on the built environment, High Performance and Optimum Design of Structures and Materials III, WIT Press, 175, pp. 177-183, 2018. 